www.jmscr.igmpublication.org Impact Factor 5.244

Index Copernicus Value: 83.27 ISSN (e)-2347-176x ISSN (p) 2455-0450 crossref DOI: _https://dx.doi.org/10.18535/jmscr/v4i12.22

\author{
Journal Of Medical Science And Clinical Research \\ IGM Publication \\ An official Publication of IGM Publication
}

\title{
The Role of Insulin Resistance in Outcome of Patients with Multi Organ Dysfunction Syndrome
}

\author{
Authors \\ Dr Ritu Gupta, Dr Sandeep Singh, Dr Nithin.R
}

\begin{abstract}
Background: Multi organ dysfunction syndrome (MODS) as the name implies, is a syndrome, not a specific disease entity, manifested by organ dysfunction affecting more than one organ. Sepsis and Multi organ dysfunction syndrome (MODS) is a common cause of mortality and morbidity. Sepsis can progress to septic shock and Multi organ dysfunction syndrome is well established as the final stage of the continuum. Sepsis and acute stress injury like MODS is an insulin resistance state and degree of insulin resistance is directly proportional to the severity of stress response.

Methodology: This was a case control study conducted in which 75 cases diagnosed to have MODS without any history of impaired glucose tolerance or other endocrine disorders were recruited and their results compared with 75 healthy controls. Fasting insulin level (FIL) and fasting blood sugar (FBS) levels were taken and Insulin resistance (IR) was calculated using HOMA. IR was compared with cases and controls and its relation with severity and outcome in patient with multi-organ dysfunction syndrome was noted and analyzed.

Observations: The result of the study showed that IR among MODS cases was $34.7 \%(n=26)$ as compared to $12 \%(n=9)$ in controls, $p(<0.001)$. The mean FBS, FIL and HOMA IR levels in cases were higher $(150.65$ $\pm 62.41,4.122 \pm 3.28$ and $1.477 \pm 1.28)$ as compared to controls $(91.43 \pm 25.67,1.68 \pm 1.35$ and $0.418 \pm 0.47)$ respectively ( $p$ value $<0.001)$. Among the cases with MODS there was $41.3 \%(n=75)$ mortality. There was no mortality in controls. The mean FBS, FIL and HOMA IR values were higher in non-survivors than survivors with $(z$ score $=2.27,5.94,4.83)$ respectively $(p$ value $<0.05)$. Among the non survivors $64.5 \%(n=31)$ had insulin resistance as compared to survivors $13.6 \%(n=44)$, association of IR was strongly positive with mortality $(p<0.001)$. Mortality was higher in $\geq 4$ organ failure cases $(60 \%)$ as compared to $<4$ organ failure cases $(32 \%)$. The mean HOMA IR value increased with the number of systems being involved (0.54,1.286,1.85 and 2.72 for 2,3,4and 5 organ involvement respectively)inferring that IR increased with severity of MODS.

Conclusion: Insulin resistance plays a important risk associated with mortality among the cases of MODS and increase in HOMA IR values is an alarming situation. Studies over a decade have shown that preventing acute hyperglycemia with insulin therapy substantially improves the outcome in critically ill patients with multi organ failure. Therefore, estimation of IR can be used as an objective tool, in patients with MODS to lessen the severity of MODS and to provide a better outcome with respect to mortality.
\end{abstract}

\section{INTRODUCTION}

The metabolic response to MODS causes rapid breakdown of the body's reserves of protein, carbohydrate, and fat leading to shift in the balance between insulin and its counter-regulatory hormones. Insulin has the inherent capability to counteract the metabolic changes observed in patients with sepsis and other critical illnesses. There is growing interest in reducing hyperglycemia in patients with acute illness. This has been 
prompted by the fact that an association between hyperglycemia and mortality is described in several studies. Studies ${ }^{(1)}$ have shown that preventing acute hyperglycemia with insulin therapy substantially improves the outcome in critically ill patients with multi organ failure. Therefore this study was undertaken to study the varied systemic involvement in MODS and the effect IR would bear in patients with MODS with respect to severity and mortality.

\section{MATERIAL AND METHODS}

This study was an observational case control study conducted at the department of General Medicine, NSCB Medical College \& Hospital, Jabalpur from March 2015 to Aug. 2016 on 75 patients and who were willing to take part in the study and gave a written informed consent and who satisfied the inclusion and exclusion criteria. The study was initiated after obtaining an ethical clearance from the institutions ethics committee.

\section{Inclusion Criteria}

- All the patients diagnosed to have multiple organ dysfunction syndrome on the basis of the following criteria :

- Physiological dysfunction and or failure of two or more organs.

- Organ failure persisting beyond 24 hours.

- Homeostasis is maintained only with intervention.

\section{Exclusion Criteria}

- Known cases of diabetes mellitus.

- Patients with family history of diabetes mellitus.

- Organ dysfunction not involving more than one system.

- Patient with history of system hypertension, coronary artery disease and metabolic syndrome.

\section{SAMPLE SIZE}

The present study was carried out on 75 patients' diagnosed with multi organ dysfunction syndrome through clinical methods and investigations admitted in Medical wards and ICU in the department of General Medicine, NSCB Medical
College \& Hospital, Jabalpur (M.P.). and 75 healthy age and sex matched controls were also taken.

\section{Criteria for selecting the cases in our study:}

All cases who fulfilled the inclusion criteria were selected. According to the definition by Baue progressive reversible dysfunction of $\geq 2$ organs from acute disruption of normal homeostasis requiring intervention, the cases of MODS were selected.

To study the various systems involved the following considerations were made:

Hepatic involvement: Cases with yellowish discoloration of urine and sclera, presence of icterus, Serum bilirubin value more than 1.2 $\mathrm{mg} / \mathrm{dl}$, SGOT more than 37U/L, SGPT more than 40U/L were taken an hepatic system involvement.

Renal involvement: Cases with decreased urine output (less than $400 \mathrm{ml} /$ day), Serum urea more than $40 \mathrm{mg} / \mathrm{dl}$ with Serum creatinine more than 1.2 $\mathrm{mg} / \mathrm{dl}$ were taken as renal system involvement.

Hematological involvement: Cases with leucopenia/leukocytosis that is less than 4000 white blood cells per micro litre or more than 11000 white blood cells per micro litre with or without thrombocytopenia (platelets less than 150 x $10^{9} / \mathrm{L}$ )

CNS Involvement: Cases with altered sensorium and Glasgow coma scale less than 13 were taken as having CNS involvement.

Respiratory system involvement: Cases having shortness of breath, tachypnea (Respiratory rate more than 20 per minute), Crepitations on auscultation, peripheral capillary oxygen saturation, ( $\mathrm{SpO} 2)$ which is an estimate of the amount of oxygen in the blood less than $85 \%$ in room air measured by pulse oximeter were taken as respiratory system involvement.

\section{PROCEDURE PLANNED}

We examined and evaluated the cases of multi organ dysfunction syndrome in the Department of Medicine, N.S.C.B. Medical College \& Hospital, Jabalpur (M.P.) from March 2015 to Aug. 2016 and insulin resistance was calculated using HOMA - IR (Homeostatic Model Assessment Insulin Resistance). 
\{Fasting plasma glucose $\}$ FFasting plasma insulin\}

HOMA-IR =

405

Following calculation of IR its correlation with severity and outcome in patients with multi organ dysfunction syndrome was assessed

The normal plasma fasting insulin levels was taken as $<25 \mathrm{mIU}^{2,3}$

The cutoff values of HOMA IR were taken as 1.45 ( \pm 1.34$){ }^{4}$

\section{DATA AND STATISTICAL ANALYSIS}

Qualitative data was represented in the form of frequency and percentage. Association between qualitative variables was assessed by Chi square test with continuity correction for all $2 \times 2$ tables and Fisher's exact test for all 2X2 tables, where pvalue of Chi square test was not valid due to small counts. Quantitative data was represented using Mean \pm SD and Median and IQR (Interquartile Range). Analysis of Quantitative data between the two groups was done using unpaired t-test if data passes 'Normality test' and by Mann- Whitney Test(or Kruscal Wallies Test for more than 2 variables) if data fails 'Normality test.' SPSS software Version 20 was used for analysis.. A 'p' value less than $0.05(\mathrm{p}<0.05)$ is considered significant.

\section{RESULTS AND OBSERVATIONS}

We enrolled 75 cases and 75 controls in our study. out of which Males constituted 60\% $(n=45)$ of total cases and $53.3 \%(n=40)$ of total controls. Females constituted $40 \%(n=30)$ of total cases and $46.7 \%(\mathrm{n}=35)$ of total controls. There was slight male preponderance but the ratio of males to females in both groups were not significant $(\chi 2=2.67 ; \mathrm{p}>0.05)$ hence comparable. Incidence of MODS was found to be more common in middle aged adults (20-50 years) $70.7 \%$. An overall slight male preponderance is seen $(60 \%$ vs $53.3 \%$ ) among cases. Incidence of IR was also found to be more common in the middle aged adults (20-50) (76.8\%). An overall slight female preponderance was seen $53.8 \%$ vs $46.1 \%$ among subjects with IR.

Among the 75 cases insulin resistance was present in $26(34.7 \%)$ as compared to controls where insulin resistance among 75 controls was present in $9(12.0 \%)$.

\begin{tabular}{|l|l|l|l|}
\hline Insulin Resistance & Case & Control & Total \\
\hline Present & $26(34.7 \%)$ & $9(12 \%)$ & $35(23.3 \%)$ \\
\hline Absent & $49(65.3 \%)$ & $66(88 \%)$ & $115(76.7 \%)$ \\
\hline Total & $75(100 \%)$ & $75(100 \%)$ & $150(100 \%)$ \\
\hline$\chi^{2}=10.77 ; \mathrm{p}<0.001$
\end{tabular}

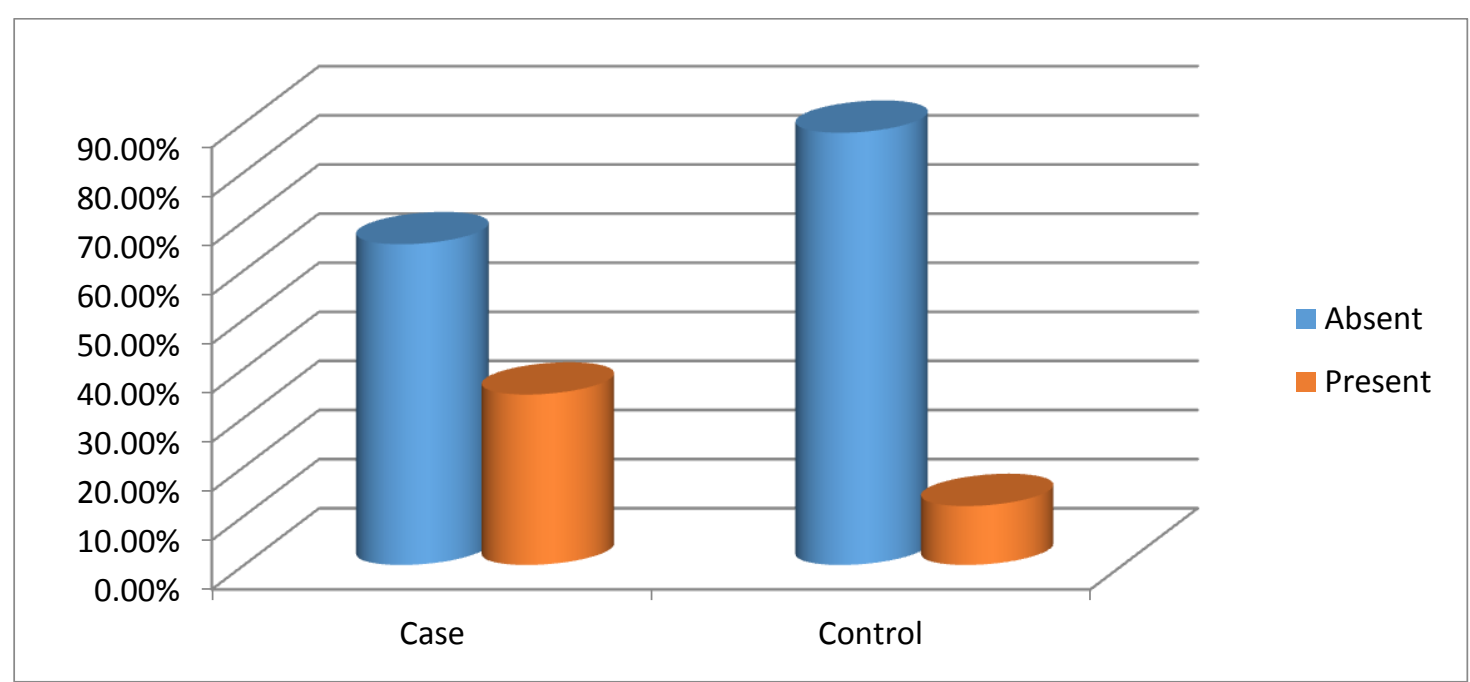


Among the 75 cases $31(41.3 \%$ ) were non survivors as compared to healthy controls where there were no non survivors.

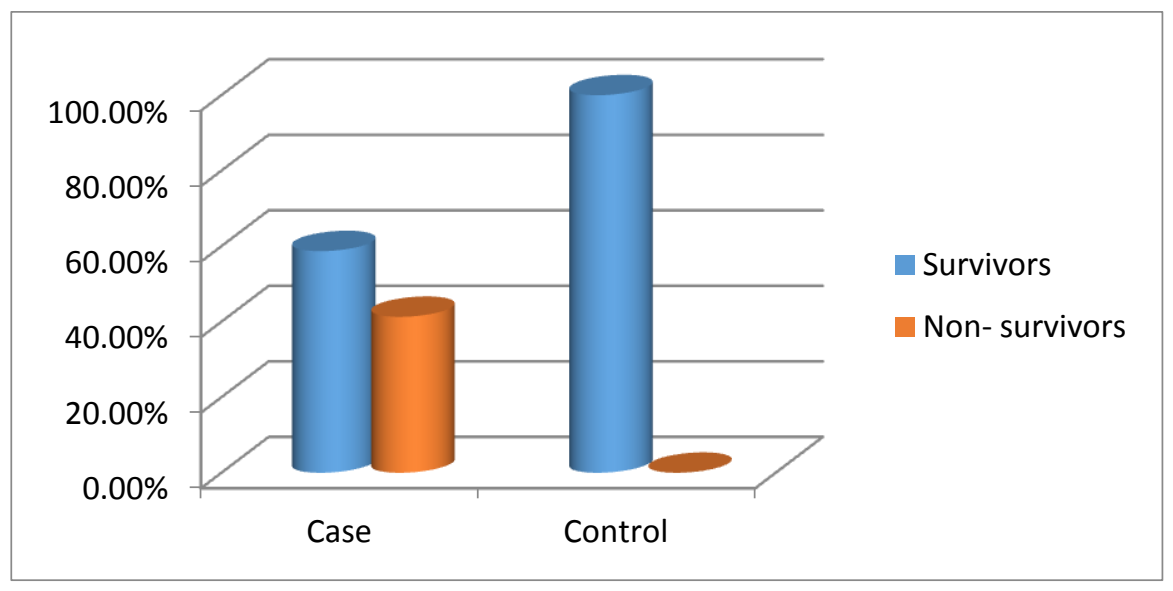

Among the 31 non survivors 20(64.5\%) of them had IR as compared 44 survivors among whom 6(13.6\%) had IR. $\left(\chi^{2}=20.79 ; \mathrm{p}<0.001\right)$

\begin{tabular}{|l|l|l|l|}
\hline Insulin resistance & survivors & Non survivors & Total \\
\hline Absent & $38(86.4 \%)$ & $11(35.5 \%)$ & 49 \\
\hline Present & $6(13.6 \%)$ & $20(64.5 \%)$ & 26 \\
\hline Total & $44(100 \%)$ & $31(100 \%)$ & 75 \\
\hline
\end{tabular}

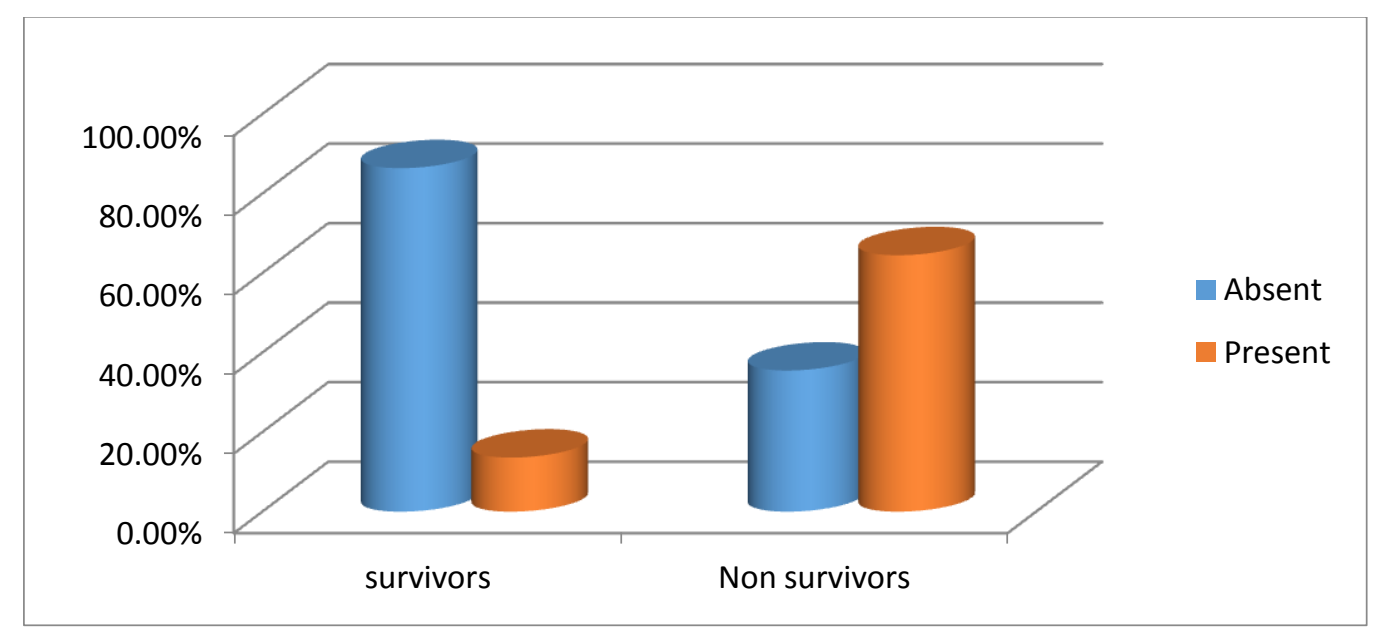

The mean FBS, Fasting insulin level and HOMA IR values were higher in cases than controls with $(\mathrm{t}=$ $7.60,5.934,6.683$ ) respectively with $\mathrm{p}$ value $<0.001$ that was significant.

\begin{tabular}{|l|c|c|c|}
\hline Variables & Cases $(\mathrm{n}=75)$ & Controls $(\mathrm{n}=75)$ & Significance \\
\hline FBS & $150.65 \pm 62.41$ & $91.43 \pm 25.67$ & $\begin{array}{c}\mathrm{t}=7.60 \\
\mathrm{p}<0.001\end{array}$ \\
\hline Fasting insulin level & $4.122 \pm 3.28$ & $1.68 \pm 1.35$ & $\begin{array}{c}\mathrm{t}=5.934 \\
\mathrm{p}<0.001\end{array}$ \\
\hline HOMA IR & $1.477 \pm 1.28$ & $0.418 \pm 0.47$ & $\begin{array}{c}\mathrm{t}=6.683 \\
\mathrm{p}<0.001\end{array}$ \\
\hline
\end{tabular}




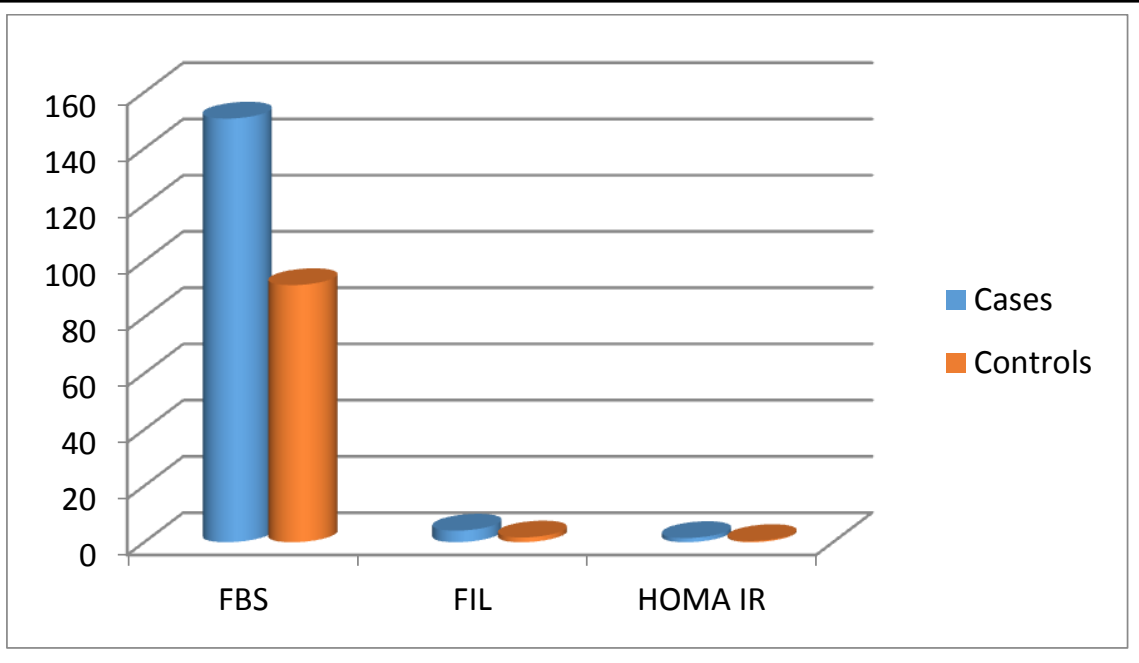

The mean FBS, FASTING INSULIN LEVEL and HOMA IR values were higher in non- survivors than survivors with $(\mathrm{z}$ score $=2.27,5.94,4.83$ ) respectively with $\mathrm{p}$ value $<0.05$ that was significant using Mann Whitney U test.

\begin{tabular}{|l|c|c|c|}
\hline Variables & survivors $(\mathrm{n}=44)$ & Non-survivors $(\mathrm{n}=31)$ & Significance \\
\hline FBS & $137.11 \pm 60.60$ & $169.87 \pm 60.79$ & $\begin{array}{c}\mathrm{Z} \text { score }=2.27 \\
\mathrm{p}<0.05\end{array}$ \\
\hline $\begin{array}{l}\text { FASTING INSULIN } \\
\text { LEVEL }\end{array}$ & $3.07 \pm 2.69$ & $5.62 \pm 3.50$ & $\begin{array}{c}\mathrm{Z} \text { score }=5.934 \\
\mathrm{p}<0.05\end{array}$ \\
\hline HOMA IR & $0.9 \pm 0.83$ & $2.24 \pm 1.38$ & $\begin{array}{c}\mathrm{Z} \text { score }=4.83 \\
\mathrm{p}<0.05\end{array}$ \\
\hline
\end{tabular}

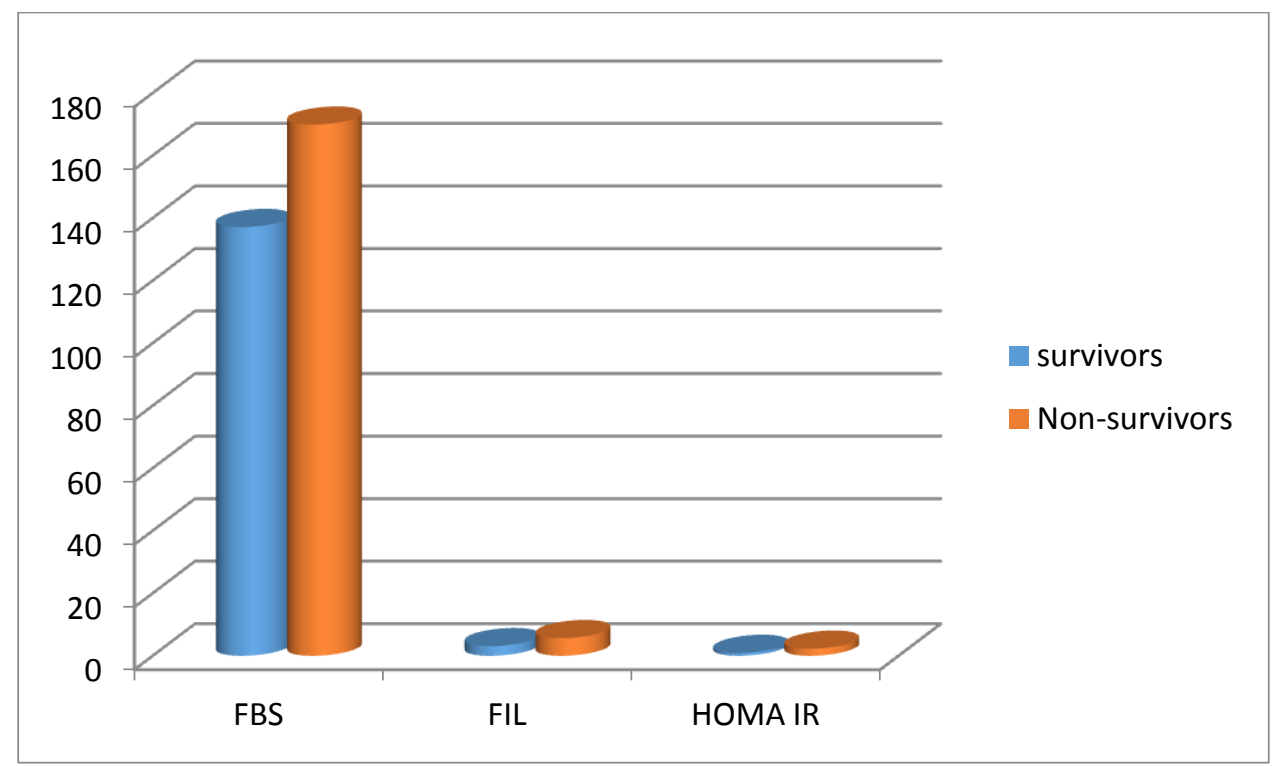

Among the various cases of MODS taken in the study renal and hematological (97.3\% each) were the most common organ failure to be followed by hepatic (70.66\%), respiratory $(36 \%)$ and neurological $(25.33 \%)$. 


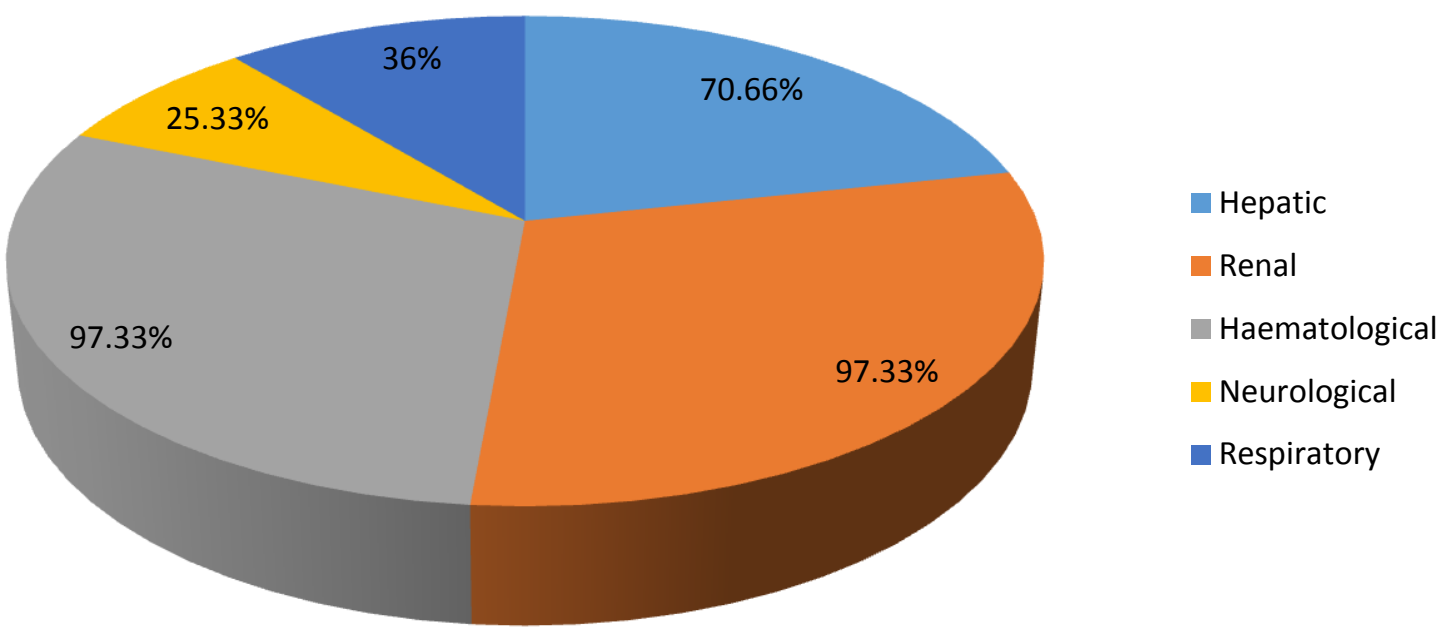

Among 50 MODS cases who had <4 system involvement $32 \%$ were non survivors. And among 25 MODS cases who $>4$ system involvement $60 \%$ were non survivors.

\begin{tabular}{|l|c|c|c|}
\hline No. of System involved & No. of Cases & Non Survivors & Total \\
\hline$<4$ & 50 & 16 & $32 \%$ \\
\hline$>4$ & 25 & 15 & $60 \%$ \\
\hline Total & 75 & 31 & \\
\hline
\end{tabular}

The Mean HOMA IR values of 2,3,4 and 5 organ system involvement were $0.54,1.286,1.85$ and 2.72 respectively. The mean HOMA IR value increased with the number of systems being involved.

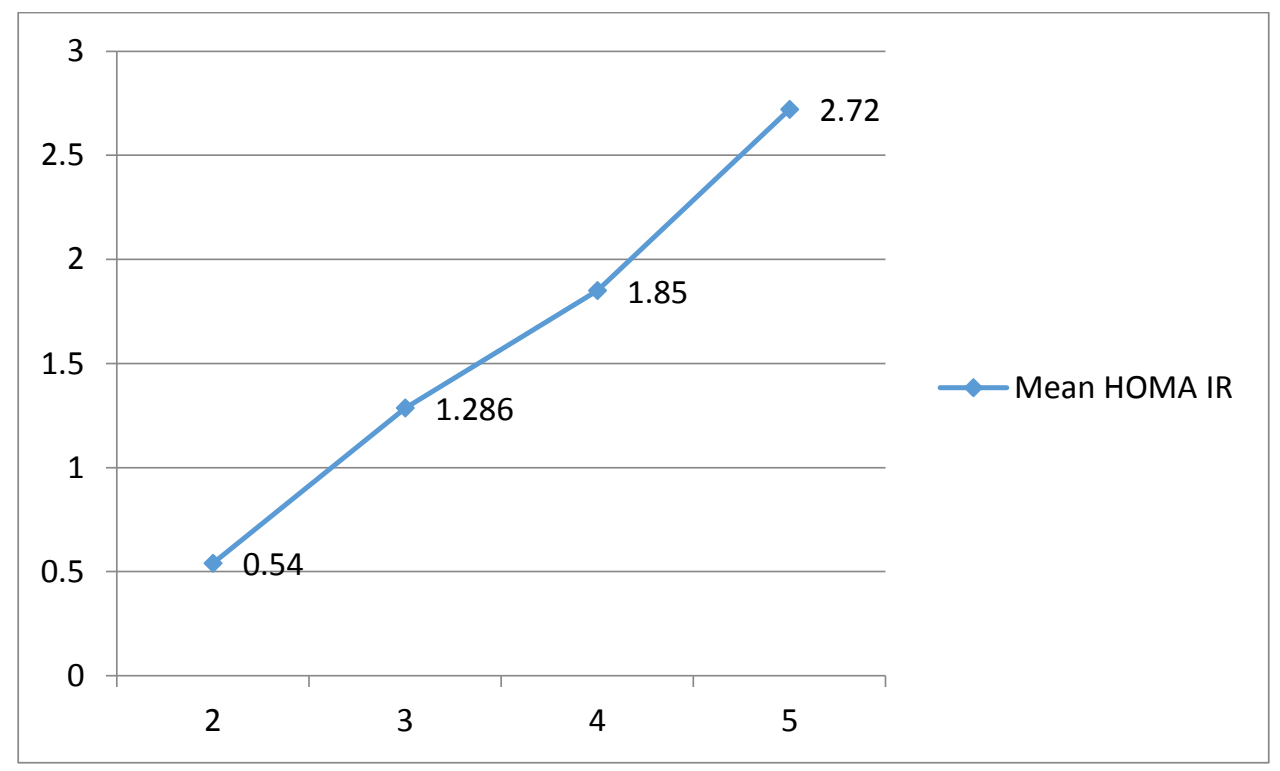




\section{DISCUSSION}

Patients with sepsis, burn, or trauma commonly enter a hypermetabolic stress state that is associated with a number of alterations in carbohydrate metabolism. These alterations include enhanced peripheral glucose uptake and utilization, hyperlactatemia, increased glucose production, depressed glycogenesis, glucose intolerance, and insulin resistance. The hypermetabolic state is induced by the area of infection or injury as well as by organs involved in the immunologic response to stress; it generates a glycemic milieu that is directed toward satisfying an obligatory requirement for glucose as an energy substrate. Hence we conducted an observational study a prospective case control study conducted at the department of General Medicine, NSCB Medical College \& Hospital, Jabalpur for a time period 1 and half years from March 2015 to Aug. 2016 on 75 patients and who were willing to take part in the study and gave a written informed consent and who satisfied the inclusion and exclusion criteria to study if insulin resistance had any role in patients with multi organ dysfunction syndrome.in our study we evaluated the various parameters and the following observations were made in relation to other studies

In our study the mean age of the patients with MODS was 38.67 years \pm 16.674 years. Incidence of MODS was found to be more common in middle aged adults (20-50 years) $70.7 \%$. An overall slight male preponderance is seen $(60 \%$ vs $53.3 \%$ ) among cases .Incidence of IR was also found to be more common in the middle aged adults (20-50) (76.8\%). It was comparable to previous studies. ${ }^{5}$

In those patients with MODS the fasting blood sugar was significantly higher as compared to controls with sugar levels being $150.65 \mathrm{mg} / \mathrm{dl}$ and $91.43 \mathrm{mg} / \mathrm{dl}$ respectively which was statistically very highly significant with $\mathrm{p}<0.001$.Fasting Insulin level in those patients with MODS was 4.1221 as compared to 1.6880 the control group which was statistically very highly significant with $\mathrm{p}<0.001$.
The mean Fasting Insulin levels in those patients with MODS was $4.122 \pm 3.28$ as compared to $1.68 \pm 1.35$ in the control group with $t=5.934$ with $p<0.001$ which was statistically very highly significant .

HOMA IR values in the study and control groups were 1.4774 and .4188 respectively which was statistically very highly significant with $\mathrm{p}<0.001$. Previous studies also showed the FBS, FIL and HOMA IR values were increased in cases with sepsis and MODS than in controls. ${ }^{6,7}$

In our study it was observed that insulin resistance was significantly higher (64.5\%) among non survivors than survivors $(13.6 \%)$ showing that patients with insulin resistance had increased risk of mortality. The values between the groups was statistically significant with chi-Square value 20.79 and $\mathrm{p}=0.000$.

Sidhartha Das ${ }^{3}$ in his study found that mean value of IR in all the 80 patients studied on the day 1 of hospitalization was $6.67 \pm 10.65$. The initial high values of serum insulin and IR were significantly reduced $(\mathrm{p}<0.05)$ as these patients recovered from their critical illness. Of those who died, the first day mean insulin levels were high (13.80 \pm $14.72 \mu / \mathrm{ml}$ as well as IR $5.14 \pm 6.76$ values) which was about similar and comparable to our study.

In our study it was observed that among cases who had $<4$ system involvement the mortality was less $32 \%$ as compared to $>4$ organ system involvement where the mortality was $60 \%$.In a study by Kyle et $\mathrm{al}^{8}$ organ dysfunction of more than or equal to 3 compared to less than 3 after adjustment for confounders was associated with intermittent hyperglycemia of more than or equal to 24 hours (OR 6.1, CI 1.8-21.2; p = 0.004). Hyperglycemia trended towards significance with mortality [3.2 (CI 0.9-11.6, p = 0.079)]. Hypoglycemia, after adjusting for the above, was not associated with mortality. Organ dysfunction ( $>$ or $=3$ versus $<3$ ) was significantly associated with hyperglycemia for $>$ or $=24$ hours. In a study by Das $\mathrm{S}$ et $\mathrm{al}^{6}$ mortality rate was highest with four and more than four organ failure cases $(21.05 \%)$. There was significantly high insulin 
resistance $(\mathrm{P}<0.05)$ in patients with stress hyperglycemia than those who were euglycemics. In our study we observed that the Mean HOMA IR values of $2,3,4$ and 5 organ system involvement were $0.54,1.286,1.85$ and 2.72 respectively. The mean HOMA IR value increased with the number of systems being involved.Thus showing that IR increased with the increase in system involvement.

\section{LIMITATIONS}

There were few limitations in our study. In our study most of the data were obtained at one time point, which was measured at the time of admission and were followed up only with respect to mortality till their stay in hospital and were not followed up with respect to repeated measurements of other variables used in the study. Because the cases in our study were taken from wards and ICU and due to lack of sophisticated instruments in our set up the scoring systems used in MODS patients could not be applied.

\section{SUMMARY}

The present study showed $34.7 \% \quad(n=26)$ of MODS patients had IR while only $12 \%(n=9)$ controls had IR.

Among the cases with MODS there was $41.3 \%$ mortality. There was no mortality in controls.

The mean FBS, FIL and HOMA IR values were higher in non-survivors than survivors with (z score $=2.27,5.94,4.83$ ) respectively with $p$ value $<0.05$ that was significant.

Among the non survivors $64.5 \%$ had insulin resistance as compared to survivors (13.6\%) which was statistically significant $(\mathrm{p}<0.001)$ showing that cases with insulin resistance had increased chances of mortality.

Among comparison of various other system involvement with insulin resistance there was significant association of hepatic $(\mathrm{p}=0.045), \mathrm{CNS}$ $(\mathrm{P}=0.014)$ and respiratory system $(\mathrm{p}=0.001)$ involvement with IR, thus showing that in cases of MODS who present with above three system involvement there is increased risk of developing insulin resistance.
It was observed that mortality was higher with 4 or more than 4 organ failure cases $(60 \%)$ as compared to less than 4 organ failure cases (32\%) In our study it was also observed that with increase in number of systems being involved there was increase in mean HOMA IR values inferring that insulin resistance increased with severity of MODS.

\section{CONCLUSION}

The prevalence of insulin resistance was significantly higher in cases of MODS than in controls. Among the various cases of MODS taken in the study renal and hematological were the most common organ failure to be followed by hepatic, respiratory and neurological .Hepatic, CNS and Respiratory system involvement had significant association with insulin resistance. The mean fasting insulin levels and fasting blood sugar levels were significantly higher in cases than in controls and also it was higher in non survivors than survivors indicating that increased levels of fasting insulin and fasting blood sugar were associated with increased chances of mortality. Our study showed that mortality was higher with 4 or more than 4 organ system involvement and the mean HOMA IR values also increased with the increase in number of systems being involved thus inferring that IR increased with severity of MODS. Studiesover a decade have shown that preventing acute hyperglycemia with insulin therapy substantially improves the outcome in critically ill patients with multi organ failure. Therefore, estimation of IR can be used as an objective tool, in patients with MODS to lessen the severity of MODS and to provide a better outcome with respect to mortality.

So we studied the systemic involvement in MODS and compared each system involvement in MODS with IR and concluded that insulin resistance increases the severity of MODS and if due care is given to the control of hyperglycemia and insulin resistance, patients can have a better outcome. Thus the present study provides a compelling rationale for such a study. 


\section{REFERENCES}

1. Van den Berghe, G., Wouters, P., Weekers, F., Verwaest, C., Bruyninckx, F., Schetz, M., Vlasselaers, D., Ferdinande, P., Lauwers, P., Bouillon, R. (2001) Intensive insulin therapy in critically ill patients. N. Engl. J. Med. 2001; 345: 1359-1367.

2. Castro A, Scott JP, GrettkeDP : Plasma insulin and glucose response of healthy subjects to varying glucose loads during three-hour oral glucose tolerance test. Diabetes, 1970;19:842-851

3. Reaven GM, Olefsky J, Farquhar JW : Does hyperglycemia or hypersumlinenimia characterize the patient with chemical diabetes? Lancet, 7763;1972:1247-11249.

4. RajatJhamb, ManormaGaiha, Al Chkravarti. DagaMK : Insulin resistance as risk factor for common carotid artery intima media thickness in essential hypertension. JIACM 2005;6(2):122-8.

5. Pilika K, Roshi E. Insulin Resistance in Early Vs Late Nutrition and Complications of Sirs in Neurosurgical Intensive Care Unit (ICU). Medical Archives. 2015 Feb;69(1):46.

6. Das S, Misra B, Roul L, Minz NT, Pattnaik M, Baig MA. Insulin resistance and beta cell function as prognostic indicator in multi-organ dysfunction syndrome. MetabSyndrRelatDisord. 2009 Feb;7(1):47- 51.

7. WU Y, WANG L, LEI S. Insulin resistance and insulin secretion in patients with severe sepsis. Clinical Education of General Practice. 2009;5:005.

8. Kyle UG, Coss Bu JA, Kennedy CE, Jefferson LS. Organ dysfunction is associated with hyperglycemia in critically ill children. Intensive Care Med. 2010;36:312-20. 